\title{
How might Generative Art be a Proposition for Cross Curricular Learning in Schools
}

\author{
Graham Newman \\ School of Communication \\ Royal College of Art \\ London, UK \\ graham.newman@network.rca.ac.uk
}

\begin{abstract}
Computing is the dominant media of our times and a cultural artifact; generative art is a subject to make art and technology relevant to young people's experiences and bring art and the Stem subjects closer together as a value proposition for Steam. Generative art promotes the value of creativity, the creative industries and smart manufacturing within education to art, design and the core science subjects. It can also address the gender gap in Stem. It makes the scientific become the organic and can be used for young people to express themselves, socially, politically and economically. Whilst there is a declining uptake in GCSE art and design, arguably being not supported by the incoming EBacc curriculum, computer science forms part of the core science subjects. Policy makers are insistent that every child learns to code, but code what? 14-17 year olds, dubbed by the media in the UK as the "Gen Z's" view learning computer science as boring, for the geeks and male dominated. Generative art is a fun and approachable way of teaching programming and fits the curriculum as an extension of existing units of enquiry.
\end{abstract}

Generative art. GCSE education. UK education policy.

\section{INTRODUCTION}

The aim of this paper is to discuss how might generative art (gen art) be a proposition for cross curricular learning in context of the current UK GCSE education. It covers the current lack of art and design focus in schools and responds to young people viewing creativity and technology as two of their key generation differentiators. Two gen art tools have been reviewed and discussed as to how might they be included as a unit of inquiry in the classroom

This research forms an early stage of the author's thesis at the Royal College of Art currently titled "Beyond Stem/Steam: UK 2020 creative practice and design education for the third industrial revolution". The intent is to look through the lens of how might design education be recast as a special branch of the sciences in order for the UKs Creative Industries to be fit for growth in context of emerging digital manufacturing markets.

The context of this paper is not a "universal" solution to other countries national education curriculums, focusing solely on the UK. It is however intended to offer in some way a roadmap of how might we as practitioners, researchers and educators prepare young people for work in the foreseeable future in which Europe can be a centre of excellence for well designed, well-thought through objects designed, manufactured and shipped through any $3 G$ device. In order to achieve this, a well prepared talent pool of artists, designers and technologists is needed. Gen art may go some way to introducing these areas of practice with the intent of a broad understanding and empathy spanning Stem, art and design.

So, what exactly is gen art and how can it be applied to learning in the classroom? Gen art is not built with plans, materials or an objective outcome. It is grown and cultivated like a tree or flower, emerging from simple functions and mathematics. The art of the creation of the organic from the logical. William Blake's painting of Newton having his back turned to the natural world in favour of "science" (his compasses) is a common interpretation of logic. Gen art can be viewed as Newton turned round; order vs chaos, which applies rather well to creative coding. Gen art can imitate nature and break linear narration creating a fluid, non-repeating world. 


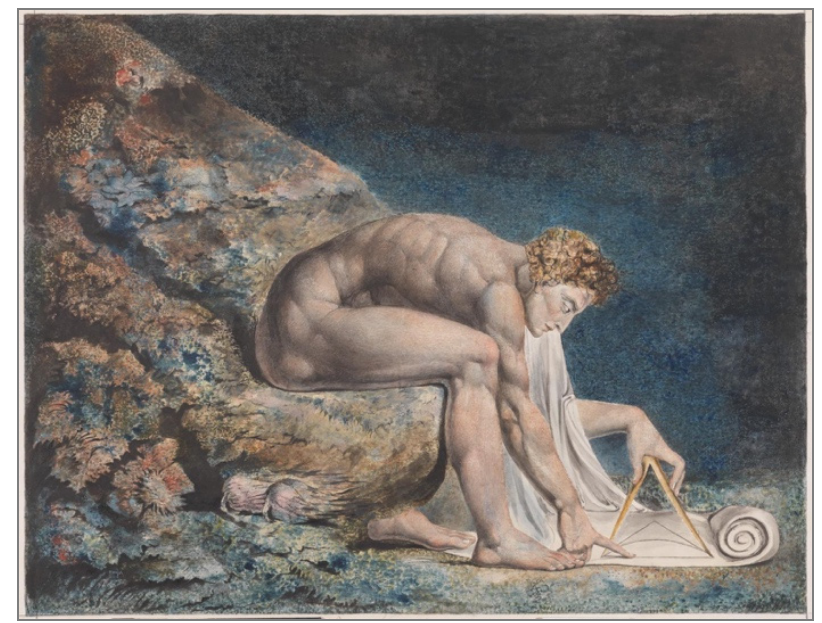

Figure 1: Newton (1795-1805) by William Blake. Collection Tate Britain.

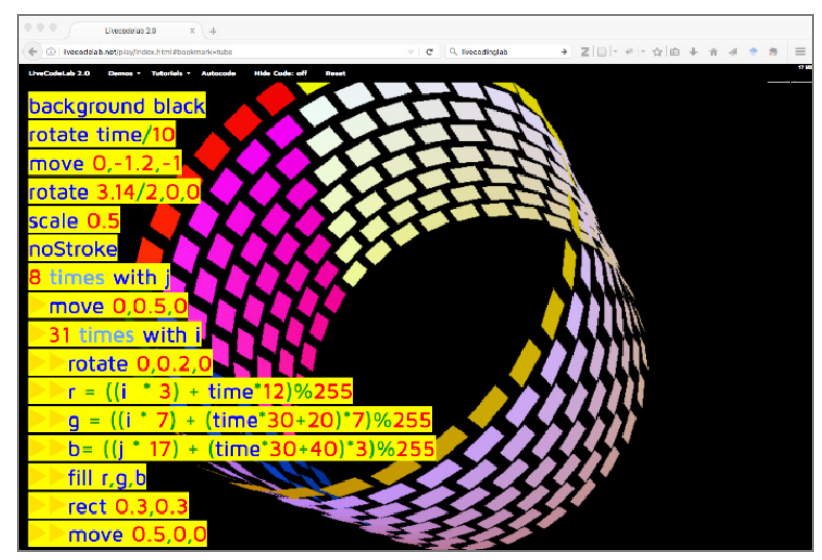

Figure 2: LiveCodeLab in action. www.livecodelab.net

To offer some background context it is worth reviewing the current general lack of satisfaction of art and design teaching, both from teachers and students in the national curriculum. Students opting for design and technology in schools at GCSE level has decreased from 440,000 in 2004 to less than 190,000 this year, an average drop of $43 \%$ (JCQ 2016). Whilst discussing this in detail is out of scope of the paper, it is of interest and part of the author's wider research to highlight the work of Bruce Archer and Ken Baynes at the RCA's Department of Design Research (DDR) and their recommendations for the then Secretary of State for Education and Science in the late 1970s - early $80 \mathrm{~s}$. What was implemented then was a watered down version of the DDR proposals and what we have currently in place is a very, very watered down version. In principal Archer et al. (1979) proposed that:

The collected experience of the material culture, and the collected body of experience, skill and understanding embodied in the arts of planning, inventing, making and doing, should be regarded as a major area of legitimate interest and activity in education at all levels.
This context of "making and doing" is central to the proposition of gen art as to somehow work in the margins between the traditional arts and sciences subjects.

\section{THE CURRENT SITUATION}

In 2014, England was the first country in the world to mandate teaching coding to children at primary and secondary schools. The introduction of computing in the national curriculum means that our school children will be taught the knowledge and skills that employers will need. (Department for Culture Media \& Sport 2017)

Ged Gast, Immediate Past President of NSEAD, claims the current curriculum is a major factor in the declining pipeline of young people needed to fill the UK's creative industries talent pool. "Owing in the main to the national standards agenda policies driven by the DfE and consequence of the removal of the Creative Diploma, reductions in applied learning, EBacc, Academisation and general disregard for the value of creativity and creative industries promoted within education in Britain at the moment. This has impacted on the numbers coming through to study and appears to be narrowing the pipeline into $\mathrm{HE}$ from schools and FE at the moment"

Government is telling us to code, but code exactly what? How do we nurture young people to visualise, think, and understand creativity coding in a world where more and more young people limit themselves to be just being passive "users" of technology and have no idea what is under the bonnet. This raises the question that the current provisions for programming and are missing the point. In short, it's a male dominated boring subject. There are many computer science undergraduates who echo this view. So how do we turn on students, from users into creative coders and digital artists who can express themselves socially politically and economically?

Young people see technology and creativity as important facets of their identity. A report by Adobe (2016) shows UK 14-17 year olds, dubbed by the media "Gen Z" learns best by doing and creating (see Table 1).

Table 1: Effectiveness of Gen Z teaching methods

\begin{tabular}{|l|c|c|}
\hline & Students & Teachers \\
\hline Doing/creating & $65 \%$ & $81 \%$ \\
\hline Watching & $56 \%$ & $56 \%$ \\
\hline Researching online & $51 \%$ & $47 \%$ \\
\hline Collaborating & $41 \%$ & $57 \%$ \\
\hline Listening & $48 \%$ & $21 \%$ \\
\hline Reading & $41 \%$ & $16 \%$ \\
\hline Writing & $37 \%$ & $24 \%$ \\
\hline
\end{tabular}


However, and reflecting the impact of the EBacc, students and teachers agree that learning is more focused around the traditional lecture format, reading and writing (see Table 2 ).

Table 2: Frequency of Gen Z teaching methods (Shown: \% All of the Time)

\begin{tabular}{|l|c|c|}
\hline & Students & Teachers \\
\hline Listening & $58 \%$ & $45 \%$ \\
\hline Writing & $54 \%$ & $63 \%$ \\
\hline Reading & $40 \%$ & $39 \%$ \\
\hline Researching online & $23 \%$ & $16 \%$ \\
\hline Doing/Creating & $15 \%$ & $29 \%$ \\
\hline Watching & $9 \%$ & $22 \%$ \\
\hline Collaborating & $14 \%$ & $21 \%$ \\
\hline
\end{tabular}

\section{TOWARDS A SOLUTION}

In January 2017 the government published its Industrial Strategy green paper which was met with muted praise. In response to this, the Creative Industries Foundation (CIF 2017) commented that the "creative industries would be one of five named sectors in the new industrial strategy was a major step forward for a sector which has never been formally recognised in a national industrial strategy before." This is a step in the right direction and one would hope that teaching technology, art and design (the basic building blocks of creative practice) should now fall into line with this policy. So why not use gen art as subject to nurture these skills. Simon Katan from the Department of Computing, Goldsmiths view is:

\footnotetext{
The teaching of generative drawing techniques make for an ideal introduction to creative programming. Learning these skills not only gives an intuitive insight into the power of computation and its expressive potential, but also brings together concepts from across the curriculum.
}

Given art is subjective, computing is objective; gen art is the point where the two should meet. We need more diverse students, better prepared students and make art feel more relevant. Gen art encourages, boys and girls to collaborate and brings the art teacher and computing teacher together.

Currently, the two best examples of gen art tools are LiveCodeLab, developed in London and p5js. LiveCodeLab is intentionally designed to be "immediately accessible to an audience with low computer literacy." (Della Casa \& John 2014). p5js is a community interested in exploring the creation of art and design with technology by using the original metaphor of a software sketchbook. Both tools can provide units of enquiry to young people interested in coding and the arts, design and technology. There is no reason why this cannot be taught from Key Stage 3 (7-9 year olds) upwards.

LiveCodeLab's roots stem from are from TOPLAP manifesto (2010) and have made significant contributions to the burgeoning "algorave" culture. The experience of writing simple lines of code and seeing results appear immediately are immensely powerful. LiveCodeLab's framework of a JavaScript engine running stunning graphic visuals and sample-based audio sequencing is a perfect example of art and science learning (the developers agonised over the choice of typeface for the front end code). The code is as much of the live performance as the real-time $3 \mathrm{D}$ visuals and music. The ability to do quick dive coding with no previous experience allows for a compelling play, learn and make experience.

The much larger $p 5 j s$ project has been developed by a community of collaborators, headed by Lauren McCarthy and funded by Processing Foundation and NYU ITP. It is JavaScript library based on the core of Processing, so basically you can do everything in Processing, now with html elements over the web, such as simulation algorithms, open data and APIs. It is an ideal platform to deliver Steam learning and offers students the ability to prepare for Higher Education subjects in either digital visual arts or computer science. McCarthy recently confirmed that the $p 5 j s$ project will be "putting more focus on the K12 level going forward".

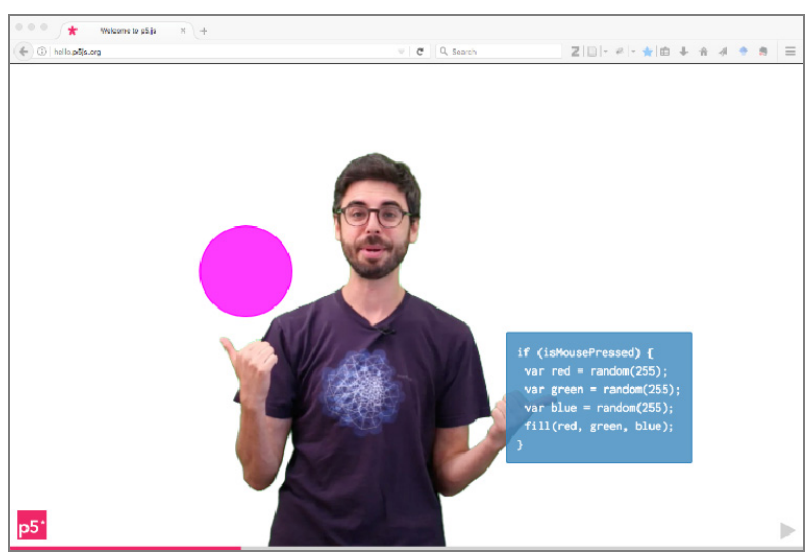

Figure 3: The unsurpassable Daniel Shiffman introducing p5js. www.hello.p5js.org

Currently only a handful of UK schools are running gen art courses. As exactly how to develop ideas and techniques for delivering this theoretical and practical content in the classroom remains to be seen. What is currently lacking in schools is a cohesive strategy between computing and art teachers. Considering only basic programming knowledge needed to create high impact, nonlinear visual art through coding, this is a great 
opportunity for Stem and the arts to work closer together. Both the logic behind the coding and the aesthetic outcomes should be reviewed for critical analysis and rigorous investigation into how the outcome was achieved, rather than examining the outcome based on a set of pre-defined parameters.

Pilot studies ran in partnership with Computing At Schools (CAS) community and Goldsmiths Creative and Digital Arts BSc course leaders in Lewisham last year revealed teaching generative art had the support of science and arts teachers, and students. Katan further adds:

Through practical and creative experimentation students can enhance their understanding on a range of topics from across the curriculum including 2D and 3D geometry, abstraction in art, Newtonian physics, evolutionary theory, probabilities and ecosystems.

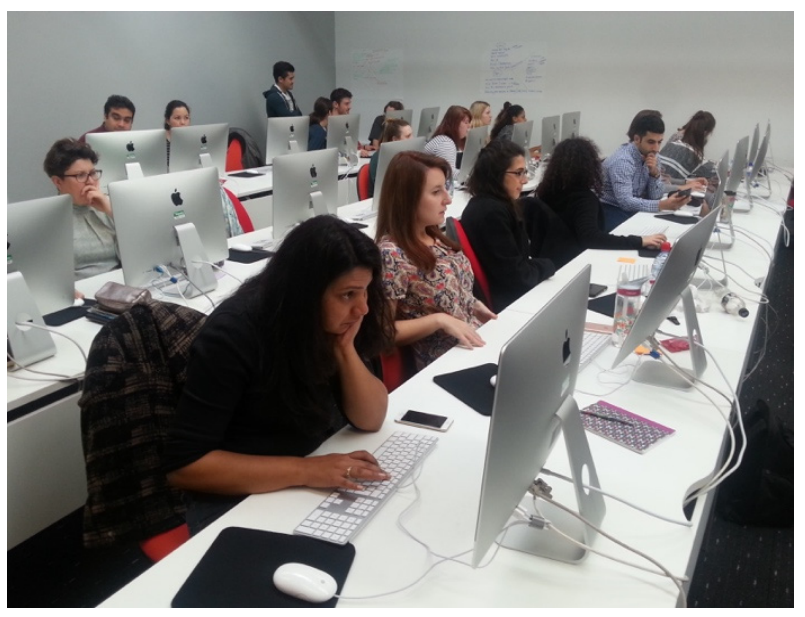

Figure 4: Primary and Secondary school teachers learning gen art at Goldsmiths.

\section{CONCLUSION}

GCSE assessments need to shift parameters and focus on the tacit knowledge learning outcomes that exist between form (of art and design) and function (of programming). This will go some way to answering the needs of future employers who will needing a workforce equipped with a hybrid skillset of art, design, technology and science: able to solve complex equations and deliver conceptual thinking. This is with reference to the Horizon 2020, the EU Framework Programme for Research and Innovation, Factories of the Future (FoF) are using digital technology to transform OEM's to retail stores. The total budget to deliver Horizon is nearly $€ 80$ billion:

What is needed is an education system fit for the 21st century. In a Brexit report from CIF published in October 2016: instead of marginalising creative subjects, we should provide young people with a mix of creative and technical skills required for success. These add to the growing clamour for a substantial change of direction in education.

This returns us to Archer and Baynes' proposition of more seeing, making and doing in the classroom. Children must be encouraged to understand how systems and their applications work through the tacit exchanges between teachers and students. Assessment should not all be outcome based on a test; the emphasis more on methods of intent, of critical inquiry and lateral thinking. Baynes (2016) still believes that "instead of marginalising creative subjects, we should provide young people with a mix of creative and technical skills required for success".

Children should experience technology as theatrical performance; the browser is a stage for creative coding; high impact, visually rich artistic content and the ability to look under the hood of technology. This environment should be encouraged in schools to incubate the next generation of artists, designers and engineers if the UK wants to remain a world leader in innovation.

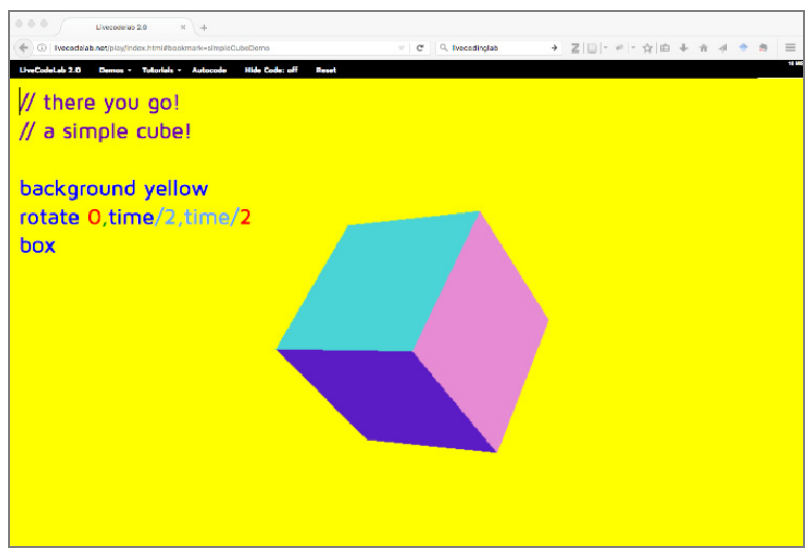

Figure 5: Background, Rotate, Box. Simple. www.livecodelab.net

The two gen art platforms discussed here can be used in the classroom immediately. They cost nothing, are open source and thus have an unlimited scope for contributions. They offer students unit of inquiry from the arts and sciences; it's the glue between the two that is of interest and consequence to all. Teachers from the arts and sciences should work closer together. None of this happens without the kinetic energy between students and motivated teachers. This month to highlight Women's day, the p5js homepage profiled Malala Yousafzai's lauded quote (as a 16-year-old) to the UN General Assembly: "Let us remember: one book, one pen, one child and one teacher can change the world". 


\section{ACKNOWLEDGEMENTS}

The author is indebted to Dr Simon Katan, Creative Computing Programme Leader, Department of Computing, Goldsmiths; Ged Gast, Immediate Past President NSEAD; Davide Della Casa and Guy John, Creative Coding Lab; Lauren McCarthy, NYU ITP; Eliza Easton Deputy Head Research and Policy, Creative industries Foundation and Ken Baynes.

\section{REFERENCES}

Adobe (2016) Gen Z in the Classroom: Creating the Future, UK Findings, October.

http://www.adobeeducate.com/genz/UK-educationgenz (accessed 1 May 2017).

Archer, B., Baynes, K., and Langdon, R. (1979) Design in General Education. Royal College of Art, April.
Baynes, K. (2016) Brexucation. Loughborough Design Press, 29 October.

http://www.Idpress.co.uk/brexucation-by-kenbaynes/ (accessed 1 May 2017).

Creative Industries Federation (2016).

http://www.creativeindustriesfederation.com (accessed 1 May 2017).

Della Casa, D. and John, G. (2014) LiveCodeLab 2.0 and its language LiveCodeLang.

JCQ (2016) Joint Council for Qualifications.

Secretary of State for Culture, Media and Sport (2017) UK Digital Strategy 2017 Policy paper, March.

TOPLAP (2010) ManifesttoDraft. https://toplap.org/wiki/ManifestoDraft (accessed 1 May 2017). 